\title{
Analogy between freezing lakes and the cosmic radiation era
}

\author{
Valerio Faraoni $\odot^{*}$ \\ Department of Physics \& Astronomy, Bishop's University, 2600 College Street, Sherbrooke, Québec, Canada J1M $1 Z 7$
}

(Received 10 December 2019; accepted 26 January 2020; published 21 February 2020)

\begin{abstract}
An equation describing a one-dimensional model for the freezing of lakes is shown to be formally analogous to the Friedmann equation of cosmology. The analogy is developed and used to speculate on the change between two hypothetical "spacetime phases" in the early universe.
\end{abstract}

DOI: 10.1103/PhysRevResearch.2.013187

\section{INTRODUCTION}

The study of the freezing of water bodies in the natural environment has a long history [1-10], appearing also in pedagogical [11-13] and popular [14] literature. The realistic problem of freezing of lakes in winter or in cold (for example, mountainous or polar) regions is difficult when factors such as the variability of atmospheric conditions, boundaries, and chemical impurities are taken into account [1-10], but it can be simplified considerably and reduced to a one-dimensional model under certain assumptions, which are best spelled out in the pedagogical literature [13]. These assumptions are that the lake covers a large area and effects at the margins can be neglected; the lake is isolated, i.e., not connected to other lakes, rivers, or bodies of water; there are no chemical impurities (the lake contains only freshwater); and the lake is deep (in practice, depths larger than $\sim 10 \mathrm{~m}$ and short periods of cold weather are considered, but this assumption can be relaxed to allow for lakes that freeze to the bottom or are permanently frozen, or for long periods of colder weather as in polar or subpolar climates or in regions at high elevation). One further neglects the geothermal input from the lake bottom, and solar radiation during the day.

The variables and parameters of the model then include [13] the ice density $\rho_{\text {ice }}$, the thermal conductivity of ice $\lambda_{\text {ice }}$, the latent heat of fusion of water $L_{f}$, the ice thickness (measured from the surface) $z$, and the water and air temperatures $T_{1}$ and $T_{3}$. The heat losses from the lake ice to the atmosphere due to convection and radiation are simplified and described by a single heat flux density linear in the difference between air and ice temperatures [13] and described by a single heat coefficient $h$. This is the main simplification of the model that makes the phenomenon tractable analytically, since both of these fluxes are in reality nonlinear and convection is notoriously difficult to model due to changing air conditions, wind, etc. We will nevertheless adopt the simplified model of Ref. [13]. In this model, equating the heat flux density

\footnotetext{
*vfaraoni@ubishops.ca

Published by the American Physical Society under the terms of the Creative Commons Attribution 4.0 International license. Further distribution of this work must maintain attribution to the author(s) and the published article's title, journal citation, and DOI.
}

from the ice surface to the atmosphere due to radiation and convection with the flux density from water to air due to conduction through the ice, a simple equation describing the time dependence of the ice thickness $z(t)$ is obtained [13]:

$$
\frac{d z}{d t}=\frac{2\left(T_{1}-T_{3}\right)}{\rho_{\text {ice }} L_{f}} \frac{1}{\frac{1}{h}+\frac{z}{\lambda_{\text {ice }}}} .
$$

For our purposes, it is convenient to rewrite this equation in a different form. By introducing

$$
\begin{aligned}
& \alpha \equiv \frac{2\left(T_{1}-T_{3}\right)}{\rho_{\text {ice }} L_{f} \lambda_{\text {ice }}}, \\
& y \equiv \frac{z}{\lambda_{\text {ice }}}, \quad y_{0} \equiv \frac{1}{h}, \\
& s \equiv y+y_{0}=\frac{z}{\lambda_{\text {ice }}}+y_{0},
\end{aligned}
$$

and squaring it, Eq. (1.1) is rewritten as

$$
\left(\frac{\dot{s}}{s}\right)^{2}=\frac{\alpha^{2}}{s^{4}} .
$$

This equation is analogous to the Friedmann equation of relativistic cosmology for a spatially flat Friedmann-LemaîtreRobertson-Walker (FLRW) universe filled with blackbody radiation, as discussed in the next section. Indeed, the Friedmann equation, which resembles an energy conservation equation for a conservative mechanical system, lends itself to analogy with equations arising in the study of many different and completely unrelated physical systems, ranging from particles in one-dimensional motion [15-19] to optical systems [20,21], condensed matter systems [22-25], the transverse profiles of glacial valleys [20,21,26], and equilibrium beach profiles [27].

\section{COSMOLOGICAL ANALOGY}

Let us recall the essential equations of FLRW cosmology in order to develop the analogy with the freezing of lakes. We follow the notation of Refs. [28,29], in which the speed of light is unity and $G$ is Newton's constant.

\section{A. FLRW cosmology: Basics}

The geometry of a spatially homogeneous and isotropic universe is necessarily given by the four-dimensional FLRW 
line element which, in comoving polar coordinates $(t, r, \theta, \varphi)$, reads

$$
d s^{2}=-d t^{2}+a^{2}(t)\left[\frac{d r^{2}}{1-K r^{2}}+r^{2}\left(d \theta^{2}+\sin ^{2} \theta d \varphi^{2}\right)\right] .
$$

In this class of solutions of the Einstein field equations of general relativity (GR), the scale factor $a(t)$ embodies the expansion history of the universe. According to the sign of the constant $K$, which describes the constant curvature of the three-dimensional spatial geometries obtained by setting $d t=0$, the FLRW line element (2.1) describes closed universes (for $K>0$ ), or Euclidean spatial sections (when $K=0$ ), or hyperbolic three-spaces (if $K<0$ ), respectively [28-31]. This classification includes all the possible FLRW geometries.

The matter content of the universe, which generates the spacetime curvature, is usually described by a perfect fluid with energy density $\rho(t)$ and isotropic pressure $P(t)$ related by an equation of state $P=P(\rho)$. The Einstein-Friedmann equations satisfied by $a(t), \rho(t)$, and $P(t)$ are [28-31]

$$
\begin{aligned}
& H^{2} \equiv\left(\frac{\dot{a}}{a}\right)^{2}=\frac{8 \pi G}{3} \rho-\frac{K}{a^{2}}, \\
& \frac{\ddot{a}}{a}=-\frac{4 \pi G}{3}(\rho+3 P), \\
& \dot{\rho}+3 H(P+\rho)=0,
\end{aligned}
$$

where an overdot denotes differentiation with respect to the comoving time $t$ and $H(t) \equiv \dot{a} / a$ is the Hubble function [28-31]. Only two of these three equations are independent: given any two, the third one can be derived from them. Without loss of generality, we adopt the Friedmann equation (2.2) and the energy conservation equation (2.4) as primary, while the acceleration equation (2.3) is derived from them.

\section{B. The analogy}

Equation (2.2) with $K=0$ is formally the same as Eq. (1.5) ruling the thickness of ice in freezing lakes if we exchange the variables $(t, s(t)) \rightarrow(t, a(t))$. The analogy holds only if the energy conservation equation is also satisfied; this happens if a suitable cosmological fluid fills the analog universe. By comparing Eqs. (1.5) and (2.2), we see that it must be

$$
\rho(t)=\frac{\rho_{0}}{a^{4}(t)}
$$

where $\rho_{0}$ is a positive integration constant determined by the initial conditions. This relation is familiar in cosmology [28-31] and in blackbody thermodynamics (e.g., [32]). More in general, if the cosmic fluid satisfies the barotropic equation of state $P=w \rho$ for a suitable constant $w$ ("equation of state parameter"), the conservation equation (2.4) integrates to

$$
\rho(a)=\frac{\rho_{0}}{a^{3(w+1)}} .
$$

Equations (2.5) and (2.6) then imply that the analogy between the freezing of lakes and cosmology holds if the analog universe is filled with blackbody radiation with equation of state parameter $w=1 / 3$ and energy density scaling as $\rho \sim a^{-4}$. The fact that the constant $\rho_{0}$ in Eq. (2.6) is

$$
\rho_{0}=\frac{3 \alpha^{2}}{8 \pi G}
$$

[as follows from Eqs. (2.5), (2.2), and (1.5)] is fortunate because it implies that the analog fluid has always positive energy density and satisfies the energy conditions. This fact cannot be taken for granted in such a far-fetched analogy between physical phenomena that are so distant from each other.

The acceleration equation (2.3) implies that the expansion of the radiation-dominated analog universe is always decelerated, $\ddot{a}<0$.

The analogy holds only if $K=0$ and

$$
s(t)=\frac{z(t)}{\lambda_{\text {ice }}}+\frac{1}{h}
$$

is analogous to the scale factor $a(t)$, while $\dot{s} / s$ is analogous to the Hubble function $\dot{a} / a$. It is straightforward to verify that the energy conservation equation and the acceleration equation are satisfied with $w=1 / 3$ and $\rho(t)=\rho_{0} / a^{4}$ if $\rho_{0}$ is as in Eq. (2.7).

The solution of Eq. (1.1) with the initial condition $z(0)=0$ is [13]

$$
z(t)=\lambda_{\text {ice }}\left[\sqrt{\frac{2 \alpha t}{\lambda_{\text {ice }}}+\frac{1}{h^{2}}}-\frac{1}{h}\right]
$$

or

$$
s(t)=\sqrt{\frac{2 \alpha t}{\lambda_{\text {ice }}}+\frac{1}{h^{2}}}
$$

with initial condition

$$
s(0)=\frac{1}{h} \equiv y_{0}
$$

(the well known square-root solution describes the radiation era of spatially flat FLRW universes filled with radiation [28-31]). Freezing begins at $t=0$ and $z=0$, corresponding to the finite value $a_{0}=1 / h$ of the analog scale factor, while the big bang $a=0$ (or $s=0$ ) corresponds to

$$
t_{0}=-\frac{\lambda_{\text {ice }}}{2 \alpha h^{2}}, \quad z_{0}=-\frac{\lambda_{\text {ice }}}{h}, \quad y=-y_{0} .
$$

It is interesting to speculate what the analogy with freezing lakes could imply if the state of the universe corresponds to a "phase of gravity" and the liquid-solid phase transition of water has some analog in gravity at the high energies and temperatures found in the early universe. In string gas cosmology, the early universe is regarded as a gas of strings and there is a phase transition at the Hagedorn temperature. Correction terms to the low-energy effective action of string theory dominate in the Hagedorn phase and, after the temperature of the universe falls below the Hagedorn temperature following the phase transition, the fundamental string states become meaningless and one has to study brane states instead (see Refs. [33,34] for reviews). There is also an extensive body of separate literature attempting to describe spacetime as an entity emerging from still unspecified building blocks ("atoms" or "molecules" of spacetime) [35-43], and another intriguing idea that has generated a large literature is the 
thermodynamics of spacetime [44-46]. In this view, it makes sense to speculate about possible phase transitions between different phases of spacetime, analogous to phase transitions in water. In the analogy, the phase change analogous to the liquid water-solid ice transition in freezing lakes begins when $a=a_{0}$; then the ice thickness grows in lakes and the universe described by GR expands to $a>a_{0}$. For lakes, it does not make sense to consider negative ice thickness $z<0$; in the cosmological analogy, the spacetime manifold view of the universe would be meaningless if $a<a_{0}$ because gravity and spacetime are in a different phase. If it carried through, this analogy would eliminate the problem of the big bang singularity at $a=0$ by stating that in the early universe [when $a(t)$ is below the critical value $a_{0}$ ] spacetime is in a different phase from the one we know today and it cannot be described by the classical Einstein-Friedmann equations. Pushing the analogy, there is no ice and $z=0$ at all times before freezing begins; this analogy leads to the idea of a nonclassical static "universe" with $a=a_{0}$ at all early times in a non-GR phase, with a static asymptotic past. This interpretation is stretched because GR would not be able to describe the early phase.

The analogy should be regarded with a grain of salt: for freezing lakes, the phase change is accompanied by a heat flux from the cooling body of water, which is clearly not isolated. The cosmological spacetime manifold, instead, is necessarily isolated, but cooling occurs because the universe expands.

\section{A SYMMETRY OF THE EQUATIONS AND OF THEIR SOLUTIONS}

A radiation fluid is a conformally invariant form of matter since the Maxwell equations are conformally invariant in four spacetime dimensions [28,29,47], and it is in principle conceivable that some form of conformal symmetry may hold. However, this is not trivial because the Einstein equations are not conformally invariant even when conformally invariant matter sources them. Nevertheless, a symmetry (vaguely) related to conformal invariance does exist and it translates in a previously unknown symmetry of Eq. (1.1) describing the freezing of lakes and of its solution.

Consider the line element of a spatially flat FLRW universe and perform a conformal rescaling of the spacetime metric $g_{a b} \rightarrow \tilde{g}_{a b}=\Omega^{2} g_{a b}$ with conformal factor $\Omega\left(x^{\alpha}\right)$. In general, the new metric $\tilde{g}_{a b}$ is not a solution of the Einstein equations with the same form of matter (when the transformed equations are rewritten as effective Einstein equations, the effective stress-energy tensor generated by the conformal transformation contains first and second covariant derivatives of $\Omega$ and does not satisfy any energy condition $[28,48]$ ). Nevertheless, some residual conformal symmetry remains, as explained below.

Using conformal time $\eta$ defined by $d t \equiv a d \eta$ [28,29], the two line elements related by the conformal rescaling $g_{a b} \rightarrow$ $\tilde{g}_{a b}=\Omega^{2} g_{a b}$ are

$$
d s^{2}=a^{2}(\eta)\left(-d \eta^{2}+d \vec{x}^{2}\right) \rightarrow d \tilde{s}^{2}=\Omega^{2} a^{2}\left(-d \eta^{2}+d \vec{x}^{2}\right) .
$$

In general, $d \tilde{s}^{2}$ is no longer a FLRW line element. However, a special situation arises if the conformal factor $\Omega$ depends only on the time coordinate $\eta$, in which case the FLRW line element remains FLRW with scale factor $\tilde{a}(\eta)=\Omega(\eta) a(\eta)$ :

$$
d \tilde{s}^{2}=\tilde{a}^{2}(\eta)\left(-d \eta^{2}+d \vec{x}^{2}\right) .
$$

However, in general this new scale factor $\tilde{a}(\eta)$ does not satisfy the Einstein-Friedmann equation with a radiation fluid. An even more special situation occurs when $\Omega=a$ : in this case [using $s(t)$ as a synonym of $a(t)$ in the rest of this work], the transformation

$$
\begin{aligned}
s & \rightarrow \tilde{s}=s^{2}, \quad s=\sqrt{\tilde{s}}, \\
d t & \rightarrow d \tilde{t}=s^{2} d t,
\end{aligned}
$$

preserves the form of the radiation fluid. In fact, we have

$$
\frac{d s}{d t}=\frac{d s}{d \tilde{t}} \frac{d \tilde{t}}{d t}=\frac{\sqrt{\tilde{s}}}{2} \frac{d \tilde{s}}{d \tilde{t}},
$$

and Eq. (1.5) becomes

$$
\frac{d \tilde{s}}{d \tilde{t}}=\frac{\tilde{\alpha}}{\tilde{s}}
$$

with $\tilde{\alpha}=2 \alpha$, i.e., it is invariant in form. The Friedmann equation is mapped into

$$
\left(\frac{1}{\tilde{s}} \frac{d \tilde{s}}{d \tilde{t}}\right)^{2}=\frac{\tilde{\alpha}^{2}}{\tilde{s}^{4}},
$$

i.e., it remains a Friedmann equation for a radiation fluid. The mapping

$$
\rho=\frac{\rho_{0}}{s^{4}} \rightarrow \tilde{\rho}=\frac{\rho}{\tilde{s}^{4}}=\frac{\tilde{\rho}}{s^{8}}
$$

leaves invariant the energy conservation equation for a radiation fluid [48]

$$
\dot{\rho}+4 H \rho=0,
$$

which is now mapped into

$$
\frac{d \tilde{\rho}}{d \tilde{t}}+\frac{4}{\tilde{s}} \frac{d \tilde{s}}{d \tilde{t}} \tilde{\rho}=0 .
$$

It is well known in the formalism of conformal transformations in FLRW space that, under the conformal transformation $\tilde{g}_{a b} \rightarrow \Omega^{2} g_{a b}$, the energy density and pressure in these spaces transforms as $\tilde{\rho}=\Omega^{-4} \rho, \tilde{P}=\Omega^{-4} P$, preserving the barotropic and constant equation of state $P=w \rho$, i.e., it is still $\tilde{P}=w \tilde{\rho}$ after the conformal rescaling. For a radiation fluid and $\Omega=a$, we have

$$
\rho \rightarrow \tilde{\rho}=\Omega^{-4} \rho=\Omega^{-4} \frac{\rho_{0}}{s^{4}}=\frac{\rho_{0}}{s^{8}}=\frac{\rho_{0}}{\tilde{s}^{4}} .
$$

To summarize, the change of variables

$$
\begin{aligned}
s & \rightarrow \tilde{s}=s^{2}, \\
d t & \rightarrow d \tilde{t}=s^{2} d t, \\
\rho & \rightarrow \tilde{\rho}=\frac{\rho}{s^{4}}=\frac{\rho}{\tilde{s}^{2}},
\end{aligned}
$$

leaves unchanged the form of the Einstein-Friedmann equations for a spatially flat, radiation-dominated, FLRW universe.

This symmetry transfers to the solution of Eq. (1.1). The solution of Eq. (1.5),

$$
s(t)=\sqrt{\frac{2 \alpha t}{\lambda_{\text {ice }}}+\frac{1}{h^{2}}},
$$


is mapped into

$$
\tilde{s}(t)=s^{2}(t)=\frac{2 \alpha t}{\lambda_{\text {ice }}}+\frac{1}{h^{2}},
$$

but we need to express this in terms of the rescaled time using $\tilde{s}(\tilde{t})=\tilde{s}(t(\tilde{t}))$. By integrating $d \tilde{t} / d t=\tilde{s}$, one obtains

$\tilde{t}=\int \tilde{s} d t=\int s^{2}(t) d t=\int\left(\frac{2 \alpha t}{\lambda_{\text {ice }}}+\frac{1}{h^{2}}\right) d t=\frac{\alpha t^{2}}{\lambda_{\text {ice }}}+\frac{t}{h^{2}}$,

where an additive integration constant has been set to zero by imposing that $t$ and $\tilde{t}$ have the same origin $t=\tilde{t}=0$. We can now solve the algebraic equation

$$
\frac{\alpha t^{2}}{\lambda_{\text {ice }}}+\frac{t}{h^{2}}-\tilde{t}=0
$$

for $t$, obtaining

$$
t(\tilde{t})=\frac{\lambda_{\text {ice }}}{2 \alpha}\left(-\frac{1}{h^{2}} \pm \sqrt{\frac{4 \alpha \tilde{t}}{\lambda_{\text {ice }}}+\frac{1}{h^{4}}}\right),
$$

and we choose the positive sign in front of the square root so that $\tilde{t}=0$ corresponds to $t=0$, consistently with what said above. Now

$$
\tilde{s}(\tilde{t})=\frac{2 \alpha t(\tilde{t})}{\lambda_{\text {ice }}}+\frac{1}{h^{2}}=\sqrt{\frac{2 \tilde{\alpha} \tilde{t}}{\lambda_{\text {ice }}}+\frac{1}{\tilde{h}^{2}}},
$$

where $\tilde{\alpha}=2 \alpha$ and $\tilde{h}=h^{2}$. In terms of the ice thickness,

$$
z(t)=\lambda_{\text {ice }}\left[s(t)-\frac{1}{h}\right]=\lambda_{\text {ice }}\left[\sqrt{\frac{2 \alpha t}{\lambda_{\text {ice }}}+\frac{1}{h^{2}}}-\frac{1}{h}\right]
$$

is mapped to

$$
\tilde{z}(\tilde{t})=\lambda_{\text {ice }}\left[\sqrt{\frac{2 \tilde{\alpha} \tilde{t}}{\lambda_{\text {ice }}}+\frac{1}{\tilde{h}^{2}}}-\frac{1}{\tilde{h}}\right],
$$

i.e., the solution is invariant in form under the symmetry operation (3.12)-(3.14).

\section{CONCLUSIONS}

The Friedmann equation (2.2) of FLRW cosmology, which is a first-order constraint on the cosmic dynamics [28] and is similar to an energy integral for a conservative system [49], lends itself to many analogies with various physical systems (usually in steady state), including particles in onedimensional motion [15-19], condensed matter systems [22-25], optical systems [20,21], glaciology [20,21,26], equilibrium beach profiles [27], and possibly other systems. Here we point out the analogy between the freezing of lakes and spatially flat, radiation-dominated FLRW universes. This analogy inspires an intriguing view of the very early universe as corresponding to an unknown phase of spacetime. If this phase is real, it cannot yet be described given our complete ignorance of the building blocks of spacetime and of the rules they obey, which could be determined by a complete theory of emergent gravity [35-43], semiclassical or corpuscolar gravity [50-56], quantum gravity, or quantum cosmology [57-60]. In the simple analogy highlighted here, the early phase of the universe would remove the problem of the big bang singularity, which would simply be inappropriate to describe with the incorrect phase of spacetime, the same way that at high temperatures it is nonsensical to talk about the properties of ice that has long been converted into liquid water or even steam.

The analogy of the freezing of lakes with cosmology uncovers a symmetry property of the relevant equation for the ice thickness and of its solution. This symmetry is completely hidden in the treatment of freezing lakes and is uncovered only thanks to a residual conformal symmetry of the analogous radiation-dominated universe in FLRW cosmology.

Another aspect of the analogy lends itself to further development: as described, the above model for the freezing of lakes necessarily contains a simplification of the complicated processes that occur in the natural environment. The simplifying conditions assumed in the model, however, can be recreated easily in the artificial, controlled environment of a laboratory. Specifically, factors such as the presence or absence of impurities in the water and their nature and concentration, the atmospheric temperature and its variation in time, the temperature at the bottom of the "lake" (in practice, a deep tank), the lack of winds, and the depth of the "lake" can all be controlled in a laboratory setting. The equipment necessary to conduct an analog gravity experiment based on the physics of water is common in cold laboratories studying snow and ice, while the equipment required is not sophisticated in comparison with that used in conventional analog gravity in which black holes, cosmological spacetimes, and curved space phenomena such as Hawking radiation, superradiance, and false vacuum decay require the use of Bose-Einstein condensates [22-25,61], ultracold atoms [62], optical systems $[63,64]$, or at least very finely controlled water flows and vortices (e.g., [65-68]). Likewise, the experimental study of the analogy between freezing lakes and cosmology would require a much simpler laboratory setting than would be necessary to study the analogy between cosmology and large geological systems such as glaciers and beaches, which also exhibit analogies with cosmology [26,27].

\section{ACKNOWLEDGMENT}

This work is supported, in part, by Bishop's University and by the Natural Sciences \& Engineering Research Council of Canada (Grant No. 2016-03803).
[1] G. Lamé and B. T. E. Clapyeron, Memoire sur la solidification par refroidissment d'un globe solid, Ann. Chim. Phys. 47, 250 (1831).
[2] J. Stefan, Ueber die theorie der eisbildung, insbesondere über die Eisbildung im Polarmeere, Ann. Phys. (Leipzig) 278, 269 (1891). 
[3] C. P. McKay, G. D. Clow, R. A. Wharton, and S. W. Squyres, Thickness of ice on perennially frozen lakes, Nature (London) 313, 561 (1985).

[4] J. C. Patterson and P. F. Hamblin, Thermal simulation of a lake with winter ice cover, Limnol. Oceanogr. 33, 323 (1988).

[5] G. D. Ashton, Thin ice growth, Water Resour. Res. 25, 564 (1989).

[6] G. E. Liston and D. K. Hall, An energy-balance model of lakeice evolution, J. Glaciol. 41, 373 (1995).

[7] D. E. Kelly, Convection in ice-covered lakes: Effects on algal suspension, J. Plankton Res. 19, 1859 (1997).

[8] J. Launiainen and B. Cheng, Modelling of ice thermodynamics in natural water bodies, Cold Reg. Sci. Technol. 27, 153 (1998).

[9] M. O. Jeffries, K. Morris, and C. R. Duguay, Lake ice growth and decay in central Alaska, USA: Observations and computer simulations compared, Ann. Glaciol. 40, 195 (2005).

[10] M. Leppäranta, Modelling the formation and decay of lake ice, in The Impact of Climate Change on European Lakes, Aquatic Ecology Series 4, edited by D. G. George (Springer, Berlin, 2010).

[11] C. F. Bohren, The freezing of streams and ponds: A simple-but uncomfortable-experiment, Phys. Teach. 42, 522 (2004).

[12] I. Soletta and M. Branca, The frozen lake: A physical model using calculator-based laboratory technology, Phys. Teach. 43, 214 (2005).

[13] M. Vollmer, The freezing of lakes in winter, Eur. J. Phys. 40, 035101 (2019).

[14] G. D. Ashton, River ice, Am. Sci. 67, 38 (1979).

[15] P. J. E. Peebles, Principles of Physical Cosmology (Princeton University Press, Princeton, 1993).

[16] W. Rindler, Relativity (Oxford University Press, Oxford, UK, 2001).

[17] T. Padmanabhan, Structure Formation in the Universe (Cambridge University Press, Cambridge, 1993).

[18] M. Szydlowski and O. Hrycyna, Dissipative or conservative cosmology with dark energy? Ann. Phys. (NY) 322, 2745 (2007).

[19] L. A. Ureña-López, Unveiling the dynamics of the universe, arXiv:physics/0609181.

[20] S. Chen, G. W. Gibbons, and Y. Yang, Explicit integration of Friedmann's equation with nonlinear equations of state, J. Cosmol. Astropart. Phys. 05 (2015) 020.

[21] S. Chen, G. W. Gibbons, and Y. Yang, Friedmann-Lemaitre cosmologies via roulettes and other analytic methods, J. Cosmol. Astropart. Phys. 10 (2015) 056.

[22] C. Barcelo, S. Liberati, and M. Visser, Analog models for FRW cosmologies, Int. J. Mod. Phys. D 12, 1641 (2003).

[23] C. Barcelo, S. Liberati, and M. Visser, Analog gravity from Bose-Einstein condensates, Class. Quantum Grav. 18, 1137 (2001).

[24] S. Eckel, A. Kumar, T. Jacobson, I. B. Spielman, and G. K. Campbell, A Rapidly Expanding Bose-Einstein Condensate: An Expanding Universe in the Lab, Phys. Rev. X 8, 021021 (2018).

[25] A. Prain, S. Fagnocchi, and S. Liberati, Analogue cosmological particle creation: Quantum correlations in expanding BoseEinstein condensates, Phys. Rev. D 82, 105018 (2010).

[26] V. Faraoni and A. M. Cardini, Analogues of glacial valley profiles in particle mechanics and in cosmology, FACETS 2, 286 (2017).
[27] V. Faraoni, Analogy between equilibrium beach profiles and closed universes, Phys. Rev. Research 1, 033002 (2019).

[28] R. M. Wald, General Relativity (Chicago University Press, Chicago, 1984).

[29] S. M. Carroll, Spacetime and Geometry: An Introduction to General Relativity (Addison-Wesley, San Francisco, 2004).

[30] A. Liddle, An Introduction to Modern Cosmology (Wiley, Chichester, 2003).

[31] E. W. Kolb and M. S. Turner, The Early Universe (AddisonWesley, Redwood City, CA, 1990).

[32] A. H. Carter, Classical and Statistical Thermodynamics (Prentice Hall, Upper Saddle River, NJ, 2001).

[33] R. H. Brandenberger, String gas cosmology, in String Cosmology, edited by J. Erdmenger (Wiley, New York, 2009), pp. 193-230.

[34] T. Battefeld and S. Watson, String gas cosmology, Rev. Mod. Phys. 78, 435 (2006).

[35] C. Barcelo, M. Visser, and S. Liberati, Einstein gravity as an emergent phenomenon? Int. J. Mod. Phys. D 10, 799 (2001).

[36] G. E. Volovik, The Universe in a Helium Droplet (Clarendon Press, Oxford, 2003).

[37] E. Verlinde, On the origin of gravity and the laws of Newton, J. High Energy Phys. 04 (2011) 029.

[38] E. P. Verlinde, Emergent gravity and the dark universe, SciPost Phys. 2, 016 (2017).

[39] S. Hossenfelder, Covariant version of Verlinde's emergent gravity, Phys. Rev. D 95, 124018 (2017).

[40] S. Liberati, Analogue gravity models of emergent gravity: Lessons and pitfalls, J. Phys.: Conf. Ser. 880, 012009 (2017).

[41] C. D. Carone, T. V. B. Claringbold, and D. Vaman, Composite graviton self-interactions in a model of emergent gravity, Phys. Rev. D 98, 024041 (2018).

[42] J. Erlich, Stochastic emergent quantum gravity, Class. Quantum Grav. 35, 245005 (2018).

[43] D. Oriti, Levels of spacetime emergence in quantum gravity, arXiv:1807.04875.

[44] T. Jacobson, Thermodynamics of Spacetime: The Einstein Equation of State, Phys. Rev. Lett. 75, 1260 (1995).

[45] C. Eling, R. Guedens, and T. Jacobson, Non-Equilibrium Thermodynamics of Spacetime, Phys. Rev. Lett. 96, 121301 (2006).

[46] T. Padmanabhan, Thermodynamical aspects of gravity: New insights, Rep. Prog. Phys. 73, 046901 (2010).

[47] J. Coté, V. Faraoni, and A. Giusti, Revisiting the conformal invariance of Maxwell's equations in curved spacetime, Gen. Relativ. Gravit. 51, 117 (2019).

[48] V. Faraoni, Cosmology in Scalar-Tensor Gravity (Kluwer Academic Press, Dordrecht, 2004).

[49] H. Goldstein, Classical Mechanics (Addison-Wesley, Reading, MA, 1980).

[50] G. Dvali and C. Gomez, Black hole's quantum $N$-portrait, Fortschr. Phys. 61, 742 (2013).

[51] G. Dvali and C. Gomez, Black hole's $1=N$ hair, Phys. Lett. B 719, 419 (2013).

[52] G. Dvali and C. Gomez, Black holes as critical point of quantum phase transition, Eur. Phys. J. C 74, 2752 (2014).

[53] A. Giusti, On the corpuscular theory of gravity, Int. J. Geom. Methods Mod. Phys. 16, 1930001 (2019).

[54] R. Casadio, A. Giugno, and A. Giusti, Matter and gravitons in the gravitational collapse, Phys. Lett. B 763, 337 (2016). 
[55] R. Casadio, A. Giugno, A. Giusti, and M. Lenzi, Quantum corpuscular corrections to the Newtonian potential, Phys. Rev. D 96, 044010 (2017).

[56] M. Cadoni, R. Casadio, A. Giusti, and M. Tuveri, Emergence of a dark force in corpuscular gravity, Phys. Rev. D 97, 044047 (2018).

[57] M. Bojowald, Quantum Cosmology: A Fundamental Description of the Universe (Springer, New York, 2011).

[58] E. Alesci, G. Botta, F. Cianfrani, and S. Liberati, Cosmological singularity resolution from quantum gravity: The emergentbouncing universe, Phys. Rev. D 96, 046008 (2017).

[59] D. Oriti, L. Sindoni, and E. Wilson-Ewing, Emergent Friedmann dynamics with a quantum bounce from quantum gravity condensates, Class. Quantum Grav. 33, 224001 (2016).

[60] J. Mattingly, Emergence of spacetime in stochastic gravity, Stud. Hist. Philos. Sci. B 44, 329 (2013).

[61] J. Braden, M. C. Johnson, H. V. Peiris, A. Pontzen, and S. Weinfurtner, Nonlinear dynamics of the cold atom analog false vacuum, J. High Energy Phys. 10 (2019) 174.

[62] J. R. Muñoz de Nova, K. Golubkov, V. I. Kolobov, and J. Steinhauer, Observation of thermal Hawking radiation and its temperature in an analogue black hole, Nature (London) 569, 688 (2019).

[63] F. D. Belgiorno, S. L. Cacciatori, and D. Faccio, Hawking Radiation: From Astrophysical Black Holes to Analogous Systems in The Lab (World Scientific, Singapore, 2019).

[64] A. Prain, C. Maitland, D. Faccio, and F. Marino, Superradiant scattering in fluids of light, Phys. Rev. D 100, 024037 (2019).

[65] S. Weinfurtner, E. W. Tedford, M. C. J. Penrice, W. G. Unruh, and G. A. Lawrence, Measurement of Stimulated Hawking Emission in An Analogue System, Phys. Rev. Lett. 106, 021302 (2011).

[66] T. Torres, S. Patrick, A. Coutant, M. Richartz, E. W. Tedford, and S. Weinfurtner, Observation of superradiance in a vortex flow, Nat. Phys. 13, 833 (2017).

[67] S. Patrick, A. Coutant, M. Richartz, and S. Weinfurtner, Black Hole Quasibound States from A Draining Bathtub Vortex Flow, Phys. Rev. Lett. 121, 061101 (2018).

[68] H. Goodhew, S. Patrick, C. Gooding, and S. Weinfurtner, Backreaction in an analogue black hole experiment, arXiv:1905.03045. 